\title{
Experimental and Calculation Studies of Main Line Oil Pumps Efficiency Change Due to Smooth Coatings
}

\author{
S. V. Akimov ${ }^{1 *}$ \\ ${ }^{1}$ TOP JSC, 8-i Eniseyskaya st., 454010 Chelyabinsk, Russian Federation
}

\begin{abstract}
The study is dedicated to investigation of impact made by smooth coatings applied on the flow part surfaces of main line oil pumps with reference to energy efficiency performance of the said pumps. Loctite 7227 and Belzona 1341 were used as coatings which allowed to significantly reduce the roughness of the coated surfaces. Distinctive features of the abovementioned coatings are low cost and easy application (by spraying or using a brush) which is, despite lack of hydrophobic behavior, makes them applicable for use in operating conditions at oil pumping stations. Experimental studies have been carried out including coatings testing on full-scale pumps MOL 1250-60 and MOL 10000-210. The results of the experimental studies have been compared with the results of analytic calculations. Based on the results of the studies the applicability of smooth non-hydrophobic coatings was confirmed on condition of their application on the surfaces of volute flow parts of mainline oil pumps. Besides, the suggested analytical procedure for coatings efficiency evaluation was validated.
\end{abstract}

\section{Introduction}

There are 2121 main line and booster pumping units currently operated in Transneft system companies. With this, the average total annual electric power consumed by main line and booster pumps equals to 14 bln $\mathrm{kWh}$ for the amount of almost 42 bln rub. which is $89 \%$ of Transneft PJSC's energy consumption [1].

It follows from the presented data that the increase in efficiency of the said pumps just for $1 \%$ will save $140 \mathrm{mln} \mathrm{kWh}$ of electric energy annually which equals to $420 \mathrm{mln}$ rub.

It is being said, that currently one of the advanced techniques for energy efficiency increase in centrifugal pumps is improving of centrifugal pump's flow part surfaces quality by applying special purpose coatings which effectiveness could be increased by means of adding extra properties to the surfaces, for example, hydrophobic properties, resistance to corrosion and erosion impact made by pumped media etc.

A reasonable amount of variety in types of coatings for pumps' flow part exist. The thesis research by S. A. Chernyshev [2] includes a comprehensive review of most of them:

\footnotetext{
*Corresponding author: akimovsv@udm.transneft.ru
} 
- cataphoresis-based coatings (epoxy mixtures application technique when a coated item is connected as a cathode in the constant electrical field of anolyte - applied material solution, and attracts cathions of the applied mixture;

- ceramic-based coatings;

- surfaces lining with various elastomeric materials (rubber and rubber-based materials);

- gas-thermal coatings based on high strength compositions, e.g. tungsten carbide-based;

- metallopolymer-based coatings (two-part polymer materials based on epoxy resins filled with fine metallic and mineral powders);

- coatings based on ionic-plasma deposition of nitrides and carbides of transition metals group IV - VI of the Mendeleev periodic system.

In addition to the above list using interface corrosion inhibitor coatings and fluoroplastic coatings is suggested in the thesis [2].

Most of the listed coatings suppose complex application techniques including inter alia a necessary use of massive on-site installations (electrocoating baths, gas-flame units, special chambers ensuring reagents circulation etc.).

Taking into account comparatively large overall dimensions of impellers and casing parts of main line oil pumps as well as the fact that the existing pumps are strewn across the vast expanse of the Russian Federation territory, it would be extremely difficult to provide an onsite application and repair of such coatings.

However, metallopolymer-based coatings among which are, for example, Loctite 7227 Nordbak Brushable Ceramic produced by Henkel (Germany) and Belzona 1341 Supermetalglide by Belzona Polymerics Limited (UK) are free from the mentioned disadvantages. These coatings are available on the market, have low cost and comparatively simple technique of application on flow part surfaces - using a brush or by spraying (the same way as common protective paint coatings are applied).

Laboratory research performed by Transneft R\&D LLC jointly with the National Research University "Moscow Power Engineering Institute" has shown that Loctite 7227 and Belzona 1341 coatings are not hydrophobic [3]. Contact angle of wetting was $<90^{\circ}$ for both coatings and close to the respective angle of the $20 \mathrm{GL}$ steel specimen.

Thus, increase of pumps efficiency when applying the mentioned coatings is ensured exclusively by reduction of surface roughness.

Decision on the abovementioned coatings practical use will require a feasibility study which in its turn makes necessary calculative and experimental study of the coatings impact on main line oil pumps' energy efficiency.

\section{Methods}

Experimental and analytical methods were adopted as research methods.

The following main line pumps manufactured by TOP JSC were chosen as the pumps under research:

- MOL 1250-260, MOL 10000-210 for studies by experimental method;

- MOL 1250-260, MOL 2500-230, MOL 3600-230, MOL 7000-210, MOL 10000-210 for studies by analytical method.

Parameters and characteristics of main line pumps are in accordance with the special technical requirements CTT-23.080.00-KTH-240-14 [4].

It should be noted that two modifications of MOL 1250-260 were tested. Modification conventionally named MOL 1250-260-1 was in production before 2017 and featured a single volute with an inlet single-vane guide unit where the vane acted as a partition of a double volute, whereas MOL 1250-260-2 modification which is currently produced features a classical double volute. 
Loctite 7227 Nordbak Brushable Ceramic and Belzona 1341 Supermetalglide were used as coating materials. In some cases, coating application was simulated by means of polishing of the corresponding flow part surfaces.

Roughness value of the coated surfaces and polished surfaces was rated as $<\mathrm{Ra} 0,8$.

Roughness value of the initial surfaces of pumps' flow part subjected to shot blasting was defined using roughness samples with the value row - Ra 12,5; Ra 25; Ra 50; Ra 100; $\mathrm{Ra} 200$, and evaluated by $\mathrm{Ra} 40$ value (standard value, intermediate value between $\mathrm{Ra} 25$ and Ra 50).

\subsection{Experimental method}

The method involves bench testing of both variants of pumps - without a coating (in an original state) and with a coating, followed by comparison of the test results.

Place of testing - TOP JSC test bench for performance testing № KNR 15-00.00.000 locates in the city of Chelyabinsk, Russia.

The test bench provides for tests performance with first class accuracy as per GOST 61342007 (ISO 9906:2007) and possesses metrological characteristics as follows:

- fractional limiting error in flow rate measurement $e_{\mathrm{Q}}=0,2 \%$;

- fractional limiting error in head measurement $e_{\mathrm{H}}=0,72 \%$;

- fractional limiting error in efficiency measurement $e_{\eta}=0,75 \%$.

Testing procedure, requirements to test bench and test conditions are in accordance with the requirements of GOST 6137-2007 (ISO 9906:1999).

The test bench design is of an open type. Testing medium - process water is kept in an underground pool with $2615 \mathrm{~m}^{3}$ capacity. Water is delivered to the tested pumps by means of two circulation vertical semi-submersible pumps operating in parallel.

Capacity control of the pumps under test is carried out by means of a control valve installed on the discharge line.

All tests have been performed at a rotor rated speed of $3000 \mathrm{rpm}$. Pump consumed power was determined as torque multiplied by rotor angular velocity. Torque measurement was carried out by a torque meter installed between the pump and the electric motor. Flow rate was measured by several electromagnetic flowmeters having different measuring ranges. A specific feature of the test bench is the possibility to obtain pump condition parameters (head, capacity, torque, efficiency, rotor speed) by means of their averaging during the set period of time (analysis interval of $60 \mathrm{sec}$ was assumed for the purpose of the study). This specific feature allows to significantly decrease the random errors of measurements caused by parameters deviation in the course of pump operation on the test bench.

Absolute values of pump parameters - capacity $Q$, head $H$, power $P$ and efficiency $\eta$ are reduced using formulas (1), (2), (3), (4) to the relative values $\varphi, \psi, \lambda, \eta_{\text {rel }}$ accordingly.

$$
\begin{gathered}
\varphi=\frac{Q}{f_{q} 2 \pi R_{2} b_{2} U_{2}}, \\
\psi=\frac{2 g H}{U_{2}^{2}}, \\
\lambda=\frac{P}{f_{q} \rho \pi R_{2} b_{2} U_{2}^{3}},
\end{gathered}
$$




$$
\eta_{\text {rel }}=\frac{\eta}{\eta_{\text {bep }}}
$$

\subsection{Analytical method}

Method of calculation of friction pressure losses in a volute was presented earlier in the work [5]. The principle of the method is integration on the volute surface of an equation (5) that connects power losses with medium flow characteristics. The equation (5), in its turn, was taken from Centrifugal Pumps by J.F. Gülich [6].

$$
\mathrm{d} P=\frac{\rho}{2}\left(c_{f}+0.0015\right) V^{3} \mathrm{~d} A
$$

where $\mathrm{d} P$ - power of losses due to fluid friction against the surface having $\mathrm{d} A$ area, $\rho$ - fluid density, $V$ - absolute speed of a fluid flow, $c_{\mathrm{f}}-$ friction factor.

Development of formulas for defining losses in a volute by integrating the expression (5) is possible provided that the volute geometry have been simplified, therefore the volute crosssectional shape is reduced to a rectangle with a constant width $B$, and the actual spiral crosssection is replaced by an equivalent Archimedes' spiral, which radius $R_{\mathrm{S}}$ and angle $\varphi$ are connected through a formula:

$$
R_{S P}=\frac{H_{\mathrm{p}}}{2 \pi} \varphi,
$$

where $H_{\mathrm{p}}$ - volute working section height.

Absolute fluid flow velocity in an arbitrary point of a volute located at a distance $R$ from the center line (pump rotor rotation axis) is defined using formulas (see also figure 1):

$$
\begin{gathered}
V=\left(V_{m}^{2}+V_{u}^{2}\right)^{0.5}, \\
V_{m}=\frac{Q}{2 \pi R B \eta_{v}}, \\
V_{u}=V_{u 2} \frac{R_{2}}{R}=\frac{g H}{\omega R_{2} \eta_{h}} \frac{R_{2}}{R}=\frac{g H}{\omega R \eta_{h}},
\end{gathered}
$$

from which we finally obtain:

$$
V=\frac{1}{R}\left(\frac{Q^{2}}{4 \pi^{2} B^{2} \eta_{v}^{2}}+\frac{g^{2} H^{2}}{\omega^{2} \eta_{h}^{2}}\right)^{0.5},
$$

where $\eta_{\mathrm{v}}, \eta_{\mathrm{h}}-$ volumetric and hydraulic efficiency accordingly. 


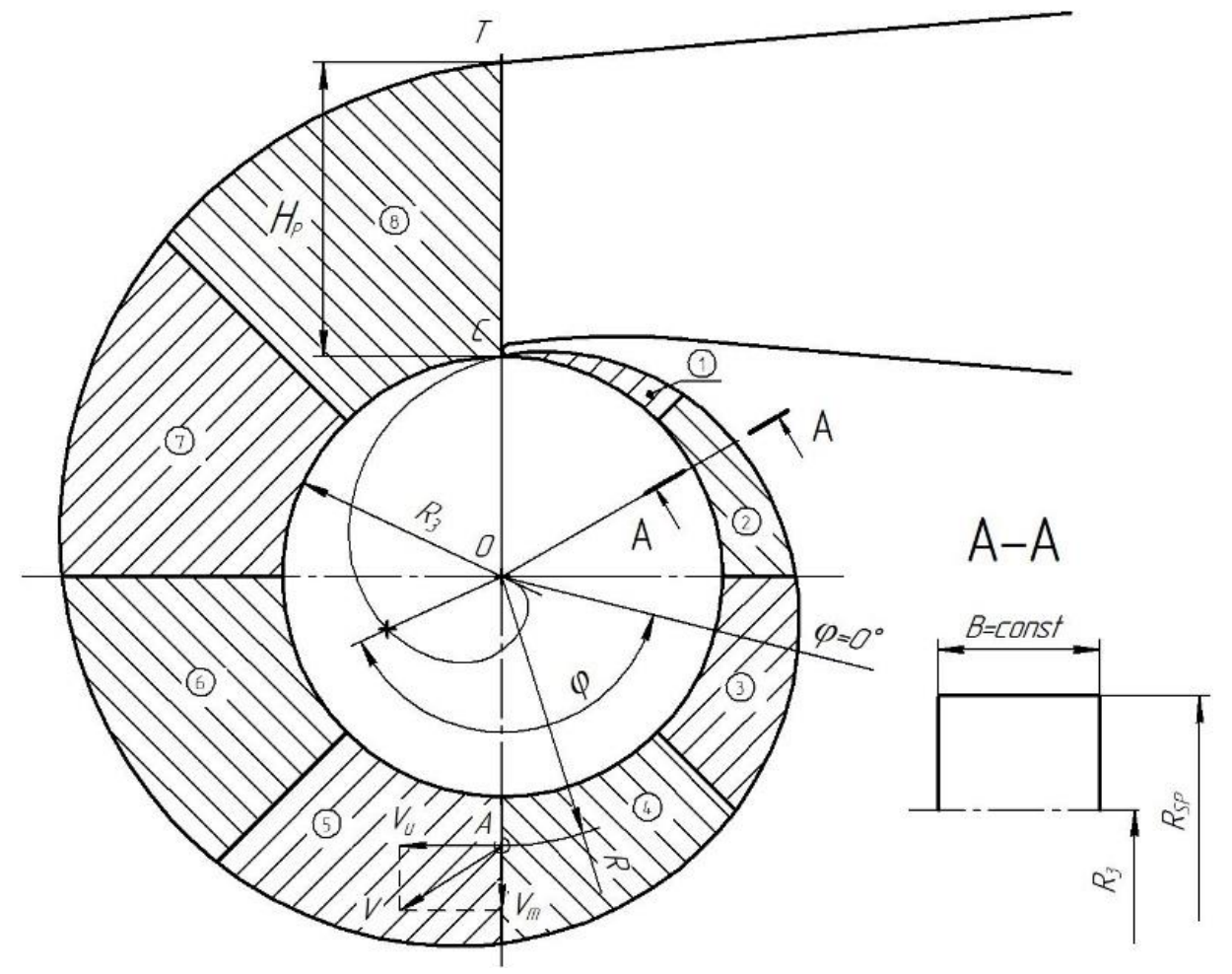

Fig. 1. Example of volute segmenting into 8 pieces.

Friction factor $c_{\mathrm{f}}$ according to [6] is defined using formula:

$$
c_{f}=\frac{0.136}{\left[-\log \left(0.2 \frac{k_{s}}{R_{h}}+\frac{12.5}{R e}\right)\right]^{2.15}},
$$

where $k_{\mathrm{s}}$ - absolute (sand) roughness, $R_{\mathrm{h}}=0,5 D_{\mathrm{h}}$ - hydraulic radius, $R e-$ Reynolds number, $v$ - cinematic viscosity of a pumped fluid.

$$
\begin{gathered}
D_{h}=\frac{4 A}{P_{w}}, \\
R e=\frac{V_{\mathrm{av}} D_{h}}{v},
\end{gathered}
$$

where $A$ - cross-sectional area of a spiral duct, $P_{\mathrm{w}}$ - duct cross-section perimeter (wetted perimeter), $V_{\mathrm{av}}$ - average velocity of flow in section.

The average value of velocity $V_{\mathrm{av}}$ is assumed as an arithmetical average of $V\left(R_{2}\right)$ and $V\left(R_{\max }\right)$, calculated using formula (10), where $R_{\max }-$ maximum radius of section (spiral wall sectional radius).

Direct integration of formula (5) is difficult due to variation in factor $c_{\mathrm{f}}$ for different sections of the volute, according to formulas (11), (12), (13). Thus, it is useful to split the volute area into several segments within the frames of which the value of $c_{\mathrm{f}}$ can be assumed constant. As a rule, 8-10 segments are enough to achieve satisfactory accuracy. 
In view of the foregoing, the result of equation (5) integration on two side walls and one spiral wall of the volute can be represented through formulas:

$$
\begin{gathered}
P=K_{1} \sum_{i=1}^{n}\left[\left(c_{f}+0.0015\right)\left(\iint_{A_{i}} \frac{1}{R^{3}} \mathrm{~d} A\right),\right. \\
K_{1}=\frac{\rho}{2}\left(\frac{Q^{2}}{4 \pi^{2} B^{2} \eta_{v}^{2}}+\frac{g^{2} H^{2}}{\omega^{2} \eta_{h}^{2}}\right)^{1.5},
\end{gathered}
$$

where $n$ - number of segments.

Integral from the formula (14) can be represented as:

$$
\iint_{A_{i}} \frac{1}{R^{3}} \mathrm{~d} A=2 I_{\mathrm{C}, i}+2 I_{\mathrm{S}, i}+I_{\mathrm{SP}, i},
$$

where $I_{\mathrm{C}}, I_{\mathrm{S}}, I_{\mathrm{SP}}-$ integrals for two side walls and one spiral wall of the volute.

$$
\begin{gathered}
I_{\mathrm{C}, i}=\frac{2 \pi}{n}\left(\frac{1}{R_{3}}-\frac{1}{R_{3}+H_{\mathrm{p}} \frac{i-1}{n}}\right) \\
I_{\mathrm{S}, i}=\left.\left[\left(2 \pi \frac{i}{n}+\frac{2 \pi R_{3}}{H_{\mathrm{p}}}\right)\left(-\frac{1}{R}\right)-\frac{2 \pi}{H_{\mathrm{p}}} \ln R\right]\right|_{H_{\mathrm{p}} \frac{i-1}{n}} ^{H_{\mathrm{p}} \frac{i}{n}} \\
I_{\mathrm{SP}, i}=-\left.\frac{2 \pi^{2} B}{H_{\mathrm{p}}^{2}}\left[\frac{1}{\varphi^{2}} \sqrt{1+\varphi^{2}}+\ln \left(\frac{1}{\varphi}\right)+\ln \left(1+\sqrt{1+\varphi^{2}}\right)\right]\right|_{2 \pi \frac{i-1}{n}+\frac{2 \pi R_{3}}{H_{\mathrm{p}}}} ^{2 \pi \frac{i}{n}+\frac{2 \pi R_{3}}{H_{\mathrm{p}}}}
\end{gathered}
$$

where $R_{3}$ - volute cutwater location radius.

In case of double volute, the latter can be considered as a system including two spiral ducts "a", "b" and one crossover duct "cd" (figure 2). 


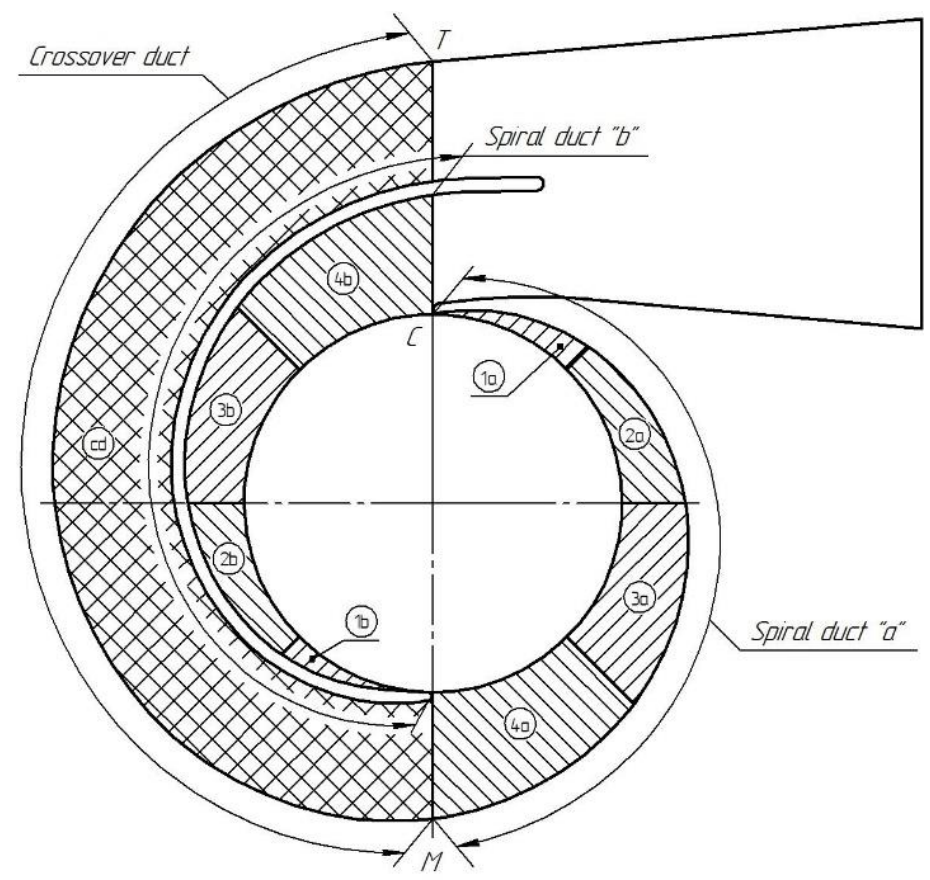

Fig. 2. Double volute.

When calculating hydraulic losses in this type of volute, to define the spiral duct losses the single volute formulas previously presented can be used, the only difference being that summation in the formula (14) is made for $\mathrm{i} / 2$ segments.

Calculation of losses in a crossover duct is also carried out using formula (5), in this case velocity is assumed constant and equal to:

$$
V_{\mathrm{cd}}=\frac{0.5 Q B}{\left(0.5 H_{\mathrm{p}}-s\right)}
$$

where $s$-partition thickness.

To calculate losses in a conical diffuser the dimensions of an equivalent circular section diffuser shall be defined whose inlet diameter equals to hydraulic diameter of the volute working section, and the length is equivalent to the length of the conical diffuser.

An equivalent diffuser, the same as the volute, shall be split into $N$ segments (figure 3 ), within the limits of which Reynolds number and hydraulic diameter are slightly changed. Thus, velocity and Reynolds number in the $\mathrm{i}^{\text {th }}$ segment can be defined using formulas:

$$
\begin{gathered}
V_{D, i}=\frac{4 Q}{\pi D_{D, i}^{2}}, \\
R e_{D, i}=\frac{V_{D, i} D_{D, i}}{v} .
\end{gathered}
$$




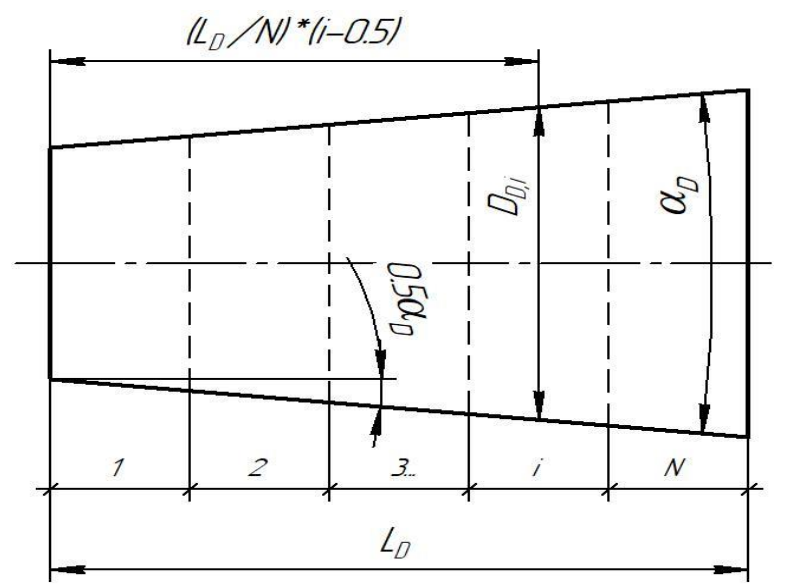

Fig. 3. Sketch of an equivalent diffuser.

Further, friction factor is calculated using formula (11) and total losses in a conical diffuser are defined using formula (23).

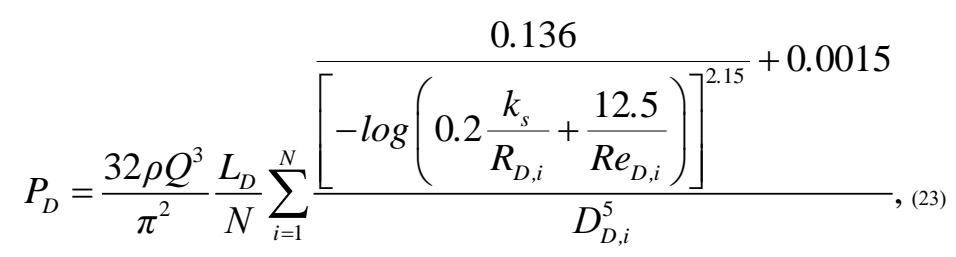

One of the main issues arising in the course of CFD-calculation and analysis aimed at defining the degree of impact of flow part surfaces quality on pump efficiency, is the issue of setting correctly the roughness value.

This issue is mainly caused by the fact that such surface characteristic as equivalent sand roughness $k_{\mathrm{s}}$ is applied in calculations whereas the results of roughness measurements on the real surfaces are usually expressed using technical values $R a$ и $R z$.

Indicator $R a$ translation into $k_{\mathrm{s}}$ can be carried out using formula (24) from the source [6]:

$$
k_{s}=\frac{6 R a}{c_{e q}},
$$

where $c_{\text {eq }}$ factor depends on the processing method and is recommended in average to be assumed 2.6.

However, depending on configuration of the surface fine irregularities, an actual value of $c_{\text {eq }}$ factor belongs to wide range - from 0.2 to 8.4 according to [6].

Therefore, validation of results when using $c_{\mathrm{eq}}=2.6$ is also an important task to be solved herein.

\section{Results}

\subsection{Results of studies via use of an experimental method}

Head and energy characteristics of MOL 1250-260-1 pump in dimensionless form, before and after coating application, are represented in figure 4 . Vertical dashed line shows the 
maximum efficiency condition and an asterisk indicates parameters of pump with a coating applied.

The coating was applied on the spiral duct surfaces and conical diffuser surfaces.

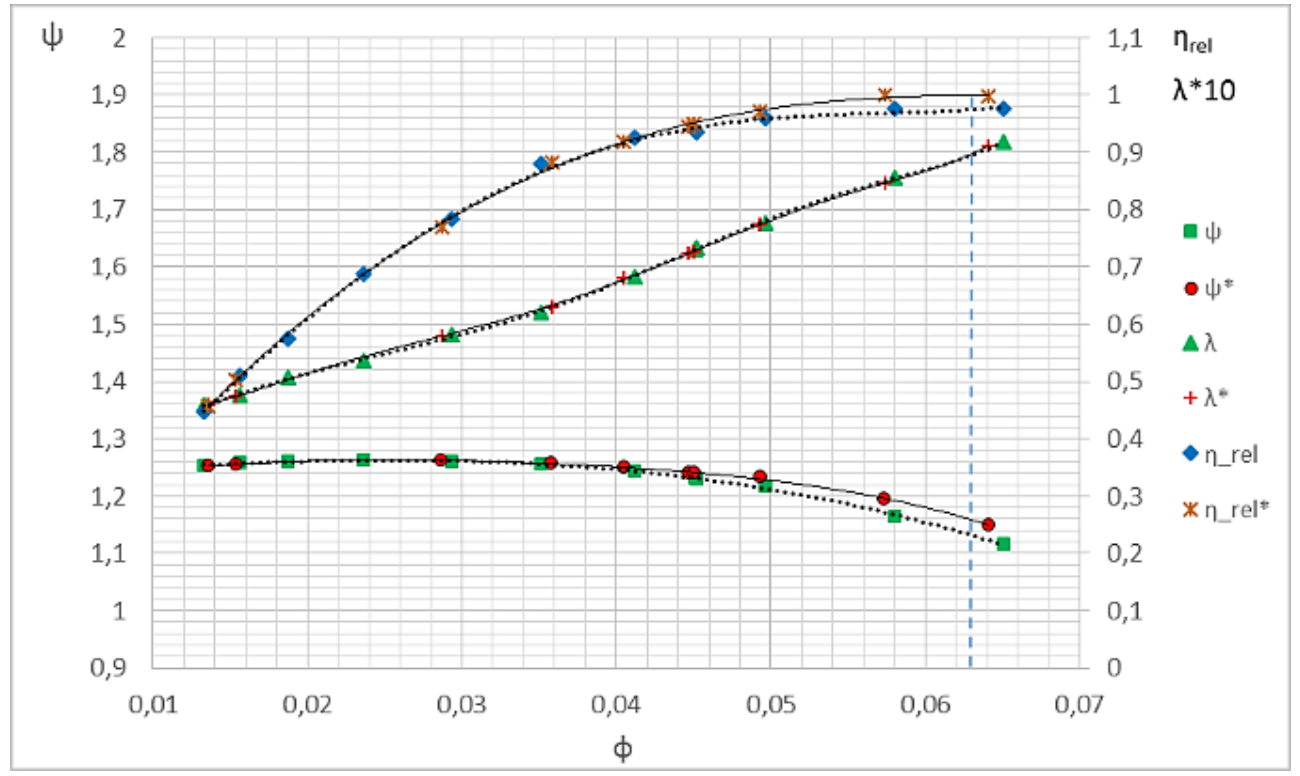

Fig. 4. Characteristics of MOL 1250-260-1.

Absence of points in the high-capacity area (to the right of dashed line) on the characteristic curves figure 4 can be explained by the fact that initially, before coating application, pump operating point, in accordance with customer's requirements, was located to the left of capacity $Q_{\text {bep }}$ (capacity at maximum efficiency) with a corresponding shift of the operating range to the left.

In this case decision on coating application on the abovementioned pump was made after it had been tested in an original state.

To modify MOL 1250-260-2 the coating application was simulated by polishing the spiral ducts of the volute.

However, the condition of the crossover duct surface as well as of the conical diffuser surface was kept original.

Head and energy characteristics of MOL 1250-260-2 in dimensionless form, before and after coating application, are given in figure 5. 


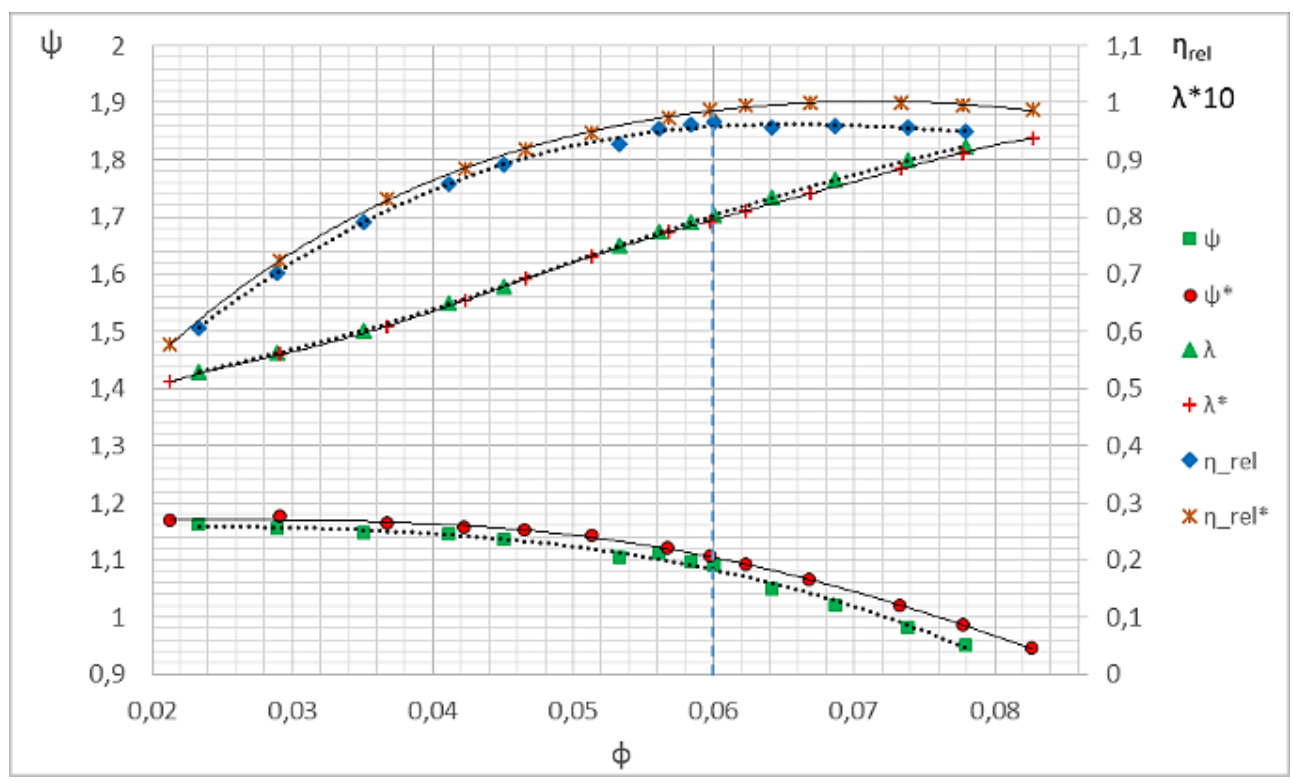

Fig. 5. Characteristics of MOL 1250-260-2.

Increase in MOL 1250-260 pumps' head and efficiency at rated capacity $\left(\sim 1250 \mathrm{~m}^{3}\right)$ equaled to:

- for MOL 1250-260-1 $-\Delta \mathrm{H}=6,7 \mathrm{~m} ; \Delta \eta=2 \%$;

- for MOL 1250-260-2 $-\Delta \mathrm{H}=4,9 \mathrm{~m} ; \Delta \eta=2.4 \%$.

Study of surface coatings of impellers installed in MOL 1250-260 pumps was not performed because of hindered access to inter-vane channels having a relatively small width and because of impracticality of coating application on impeller disks due to high quality of their original surfaces $(\mathrm{Ra}<1.6)$.

Similar experimental research was carried out for MOL 10000-210 pump. As in the case of MOL 1250-260-2 the spiral ducts of the volute were polished. Tests with coating of impeller vanes showed unreliability of such coating. During inspection of impellers of two MOL 10000-210 pumps significant coating chipping was revealed on the vanes' surfaces which, apparently, can be explained by a hindered access to inter-vane channels and therefore impossibility to ensure high quality application of the coating. In this case mechanical polishing of vanes seems to be useful as well as choosing corrosion-resistant materials for impellers manufacture.

Test results of MOL 10000-210 pump with polished surfaces of the volute spiral channels have practically coincided with the results of the initial variant.

Slight increase of efficiency for $0.1 \ldots 0.2 \%$ had been observed in the area of its maximum values which cannot be considered as a valid result because the limit error for efficiency measurement $e_{\eta}=0.75 \%$ on the test bench significantly exceeds the mentioned values.

Difference between the result obtained for MOL 10000-210 and the result obtained for MOL 1250-260 pumps can be explained by the fact that dimensions of channels' crosssections (hydraulic diameters of channels) in MOL 10000-210 are considerably larger than those of MOL 1250-260 pumps.

At the same time, the absolute roughness value of flow part cast surfaces on both pumps is Ra40 because this value is mainly defined by casting techniques. Therefore, relative roughness value (absolute roughness - hydraulic diameter ratio) of the original flow part 
surfaces of MOL 10000-210 are considerably less than that of MOL 1250-260, and reduction of relative roughness while coating application is not so dramatic.

The accuracy of defining MOL 10000-210 efficiency increase value by means of CFD analysis is also doubtful because CFD method can have its error exceeding $0.2 \%$.

In the light of the above the impact of coatings applied on a volute on pumps efficiency is advisable to study by analytical method which lacks the disadvantages of the experimental method and CFD method in terms of errors.

\subsection{Results of studies via use of an analytical method}

With reference to roughness $k_{\mathrm{s}}=92 \mu \mathrm{m}(\mathrm{Ra} 40)$ and $k_{\mathrm{s}}=0 \mu \mathrm{m}$ efficiency increase values were obtained for MOL type pumps by comparative calculations using an analytical method (figure 6).

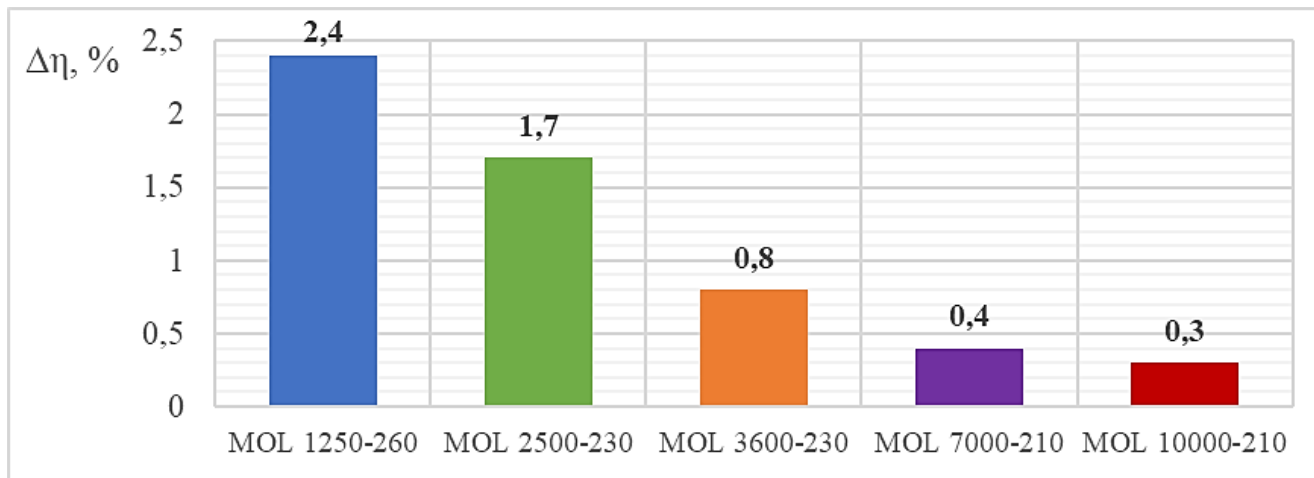

Fig. 6. Efficiency increases in MOL pumps.

It is notable that for MOL 1250-260-2 coincidence of calculated and experimental values was $100 \%$. Such accurate result is most probably accidental because similar calculations carried out for MOL 1250-260-1 pump where the coating was applied not only on spiral channels but also on the crossover duct with a conical diffuser showed efficiency increase for $2.2 \%$ in contrast to experimental value $2.0 \%$ which is, however, also a good result.

As expected, efficiency increase for MOL 10000-210 was a small value of $0.3 \%$ which is extremely difficult to record adequately by experimental methods.

Values of decrease in MOL pumps consumed power corresponding to the abovementioned efficiency increase values shall be additionally analyzed.

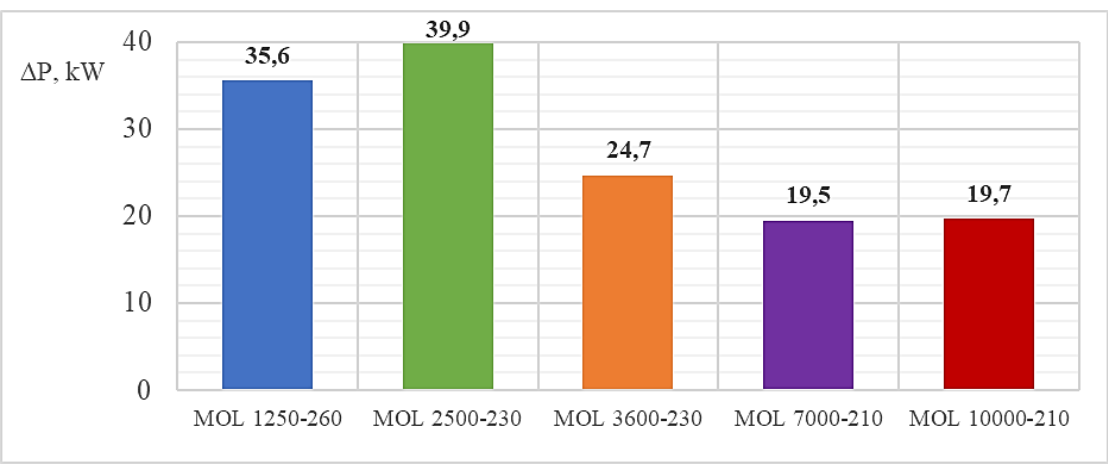

Fig. 7. Decrease in power consumption by MOL pumps. 
As it can be seen from figure 7 the decrease in power consumption for all pumps is a significant value.

\subsection{Estimation of cost advantage of smooth coatings application}

Considering the obtained power consumption decreases values it is possible to estimate annual money savings. For this purpose, we can assume the cost of one kilowatt-hour of electric energy equal to 4 ruble and the pump annual load factor (pump annual running hours - number of hours in a year ratio) $\mathrm{kR}$ equal 0.5 .

Annual average money savings can be defined using formula:

$$
S_{\mathrm{av}}=8760 \cdot k_{R} \cdot k_{\rho} \cdot \Delta P \cdot C_{e e}
$$

where $C_{e e}$ - cost of one kilowatt-hour of electric energy, $k_{\rho}$ - factor considering the density of pumped medium (medium density - water density ratio, for oil $k_{\mathrm{p}}=0.86$ ), 8760 - number of hours in a year.

Considering that $1 \mathrm{~kg}$ of LOCTITE 7227 Nordbak Brushable Ceramic costs approximately 8.3 thousand rubles in the year 2019 and $1 \mathrm{~kg}$ of this coating is enough to apply on $100 \%$ of spiral channels area of MOL 1000-210 volute, it is evident that the annual cost advantage will practically correspond with the values given in figure 8 .

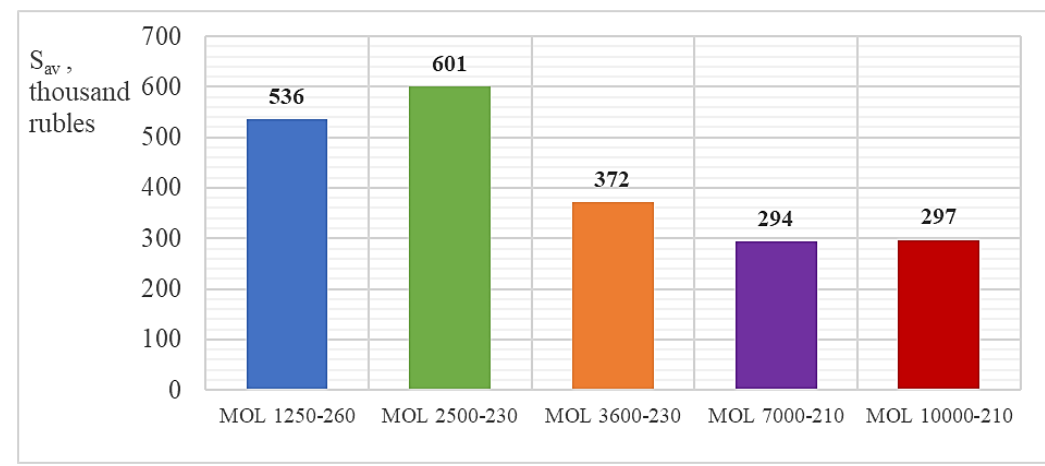

Fig. 8. Average annual savings, thousand rubles.

Trial operation of MOL 1250-260-1 pump with Belzona 134 coating applied started in 2018 at an oil pumping station. At the last pump stripping in October 2019 its running hours equaled to more than 4000 hours ( 0,46 of one year duration) for 1 year and a half. The coating was in a satisfactory condition, there were no coating chipping or wear found on the spiral channel surfaces. Thus, it can be stated with high degree of certainty that the abovementioned cost advantage will be guaranteed at $k_{\mathrm{R}}=0.5$.

\section{Discussion}

Significant effect - reduction of power consumption by one pump within the range of values from 20 to $40 \mathrm{kWt}$ as well as cost advantage from 300 to 600 thousand rubles per year can be achieved even if Loctite 7227 Nordbak Brushable Ceramic and Belzona 1341 Supermetalglide coatings lack of hydrophobic properties. With this, efficiency increase values at rated capacity fall within the range from $2.4 \%$ for MOL $1250-260$ to $0.3 \%$ for MOL 10000-210.

However, several researchers, for example [2], note that having hydrophobic properties can considerably enhance the coating effect. Hence, it follows that coatings manufacturers 
should lean toward development of new compositions combining the advantages of both smooth non-hydrophobic and hydrophobic coatings:

- easy application on surfaces;

- repairability of coatings;

- low cost;

- additional decrease in hydraulic losses due to hydrophobic properties.

Considering the quantity of pumps operated in Transneft system (more than $2000 \mathrm{pcs}$ ) potential savings can amount to 600 million rubles and more per year.

Though the cost advantage values are high, there is still an additional potential for its growth. This potential can be fulfilled provided that the coating will be applied on crossover channel surfaces of a double volute and on conical diffuser surfaces.

In the design of MOL pumps currently in use the crossover channel surfaces are not accessible for paint coating and there is no access to the conical diffuser surface for coating repair in the course of pump operation because MOL pump branches are connected to pipelines by welding.

Access to the said surfaces can be provided through improvement of pump design, for example, by equipping pumps with detachable disassemblable flow parts as it is implemented in the utility model patent RU193781U1 (Single Stage Double Entry Centrifugal Pump).

The mentioned area of work is a promising one and requires research and development activities.

Agreement of experiment results with the results of efficiency increase calculations carried out by using the analytical method suggested herein shows the validity of the approach. The mentioned agreement also confirms feasibility of using formula (23) for technical roughness value $R a$ translation into an equivalent sand roughness value $k_{\mathrm{S}}$ for cast surfaces exposed to shot blasting. The suggested analytical procedure can be used for express analysis of coatings effectiveness at pumps operation in the peak efficiency region as well as in cases where efficiency increase values are too low and cannot be adequately defined by using other methods (experiment, CFD calculations).

Pump characteristics behavior after coating application was defined using an experimental method:

- efficiency increase occurs mainly due to increase in pump head;

- efficiency increase is bigger when pump operates at capacities exceeding the rated one, and declines at capacities lower than the rated one;

- coating application results in shift of capacity value corresponding with peak efficiency to the side with higher values (to the right of the characteristic curve).

It should be additionally noted that validation of CFD methods and the respective software from the point of view of its use for coatings effectiveness evaluation is a promising field of work.

\section{Conclusion}

Experimental study of smooth coatings impact on the efficiency of main line oil pumps have been carried out.

Practical evaluation of an analytical procedure for coatings effectiveness assessment have been performed; based on the results of the evaluation a conclusion about the validity of the said procedure was made.

Based on the results of performed studies the feasibility of using smooth coatings of Loctite 7227 Nordbak Brushable Ceramic and Belzona 1341 Supermetalglide type for application on volute flow parts in all types of MOL pumps was validated. 
Estimation of cost advantage have been carried out which amounted from 300 to 600 million rubles per year for one pump depending on its type and size.

\section{References}

1. P. A. Revel-Muroz, Development of methods for improvement of oil transportation energy efficiency with introduction of a complex of energy saving technologies. Thesis in support of candidature for a technical degree (Ufa) pp 11-12, 2018

2. S. A. Chernyshev, Improvement of centrifugal pumps performance through change of work flow hydrodynamic interaction with flow part elements. Thesis in support of candidature for a technical degree (Moscow) pp 38-44, 2008

3. V. I. Voronov, Kazantsev M. N., Flegentov I. A., Starshinov D. M., Ivanov A. G. Testing of hydrophobic coatings applied on pumps' flow parts aimed at improvement of main line oil pumps efficiency. Summary scientific and technical report (Moscow: Transneft R\&D, LLC) p 43

4. Oil and Oil Products Main Pipeline Transportation. Advanced Main Line and Booster Pumps. Special Technical Requirements. CTT-23.080.00-KTH-240-14 (with Amendment 1). (Moscow: Transneft R\&D, LLC) pp 17-18

5. S. V. Akimov and Shoter P. I. A new approach for calculating hydraulic losses in centrifugal pump's volute J. Pumps. Turbines. Systems 26 56-65, 2018

6. J. F. Gülich, Centrifugal pumps (Villeneuve, Switzerland: Springer International Publishing), 2020 